\title{
Initial Study of Neuromorphic Application for Vision-Based Navigation
}

\author{
By Kazuki KARIYA $^{1)}$ and Seisuke FUKUDA ${ }^{2)}$ \\ ${ }^{1)}$ Department of Space and Astronautical Science, SOKENDAI, Sagamihara, Japan \\ ${ }^{2)}$ Institute of Space and Astronautical Science, JAXA, Sagamihara, Japan
}

(Received July 12th, 2019)

\begin{abstract}
High precision landing capability increases accessibility to scientifically interesting areas and makes a safe landing. Vision-based navigation is one of the GN\&C functions to achieve that. The navigation technique requires onboard and real-time image processing algorithms performing image recognition, feature extraction, matching, tracking, etc. However, despite the demand for fast image processing task, space-grade computers have been slower than commercial ones, lagging behind them by 1-2 orders of magnitude in performance. To address the issue, we study the applicability of a vision-based navigation algorithm for crater classification in architecture of neuromorphic processor chips which are expected to operate with ultra-low power consumption. The neuromorphic processor chip operates asynchronously and in parallel mimicking neuro-biological architecture like our brain. Therefore, the processor operates as a spiking neural network (SNN). The difference in mechanisms between timing-based spiking neurons and rate-based artificial neurons has a problem of how to make the SNNs with equivalent functions as artificial neural networks (ANNs). This study proposes a method to train the SNN by transferring weights of the ANN trained specially as synaptic efficacy of the SNN, and also evaluates the accuracy and power consumption of the SNN.
\end{abstract}

Key Words: Terrain Relative Navigation, Neuromorphic Computing, Spiking Neural Network

$\begin{array}{cll}\text { Nomenclature } & & \\ b & : & \text { bias } \\ E & : & \text { total energy } \\ I & : & \text { input current } \\ S & : & \text { spike train } \\ t & : & \text { time } \\ V & : & \text { membrane potential } \\ V_{t h} & : & \text { membrane threshold } \\ W & : & \text { convolutional kernel } \\ w & : & \text { weight parameter } \\ x & : & \text { activation of neuron } \\ \alpha & : & \text { energy consumption } \\ \eta & : & \text { firing rate } \\ \theta & : & \text { synaptic efficacy } \\ \sigma & : & \text { standard deviation } \\ \tau_{r} & : & \text { refractory period } \\ \tau_{s} & : & \text { synaptic time constant } \\ \tau_{m} & : & \text { membrane time constant } \\ i, j & : & \text { Index of neurons } \\ k & : & \text { Index of convolutional kernels }\end{array}$

\section{Introduction}

High precision landing capability is an important technique to be acquired because it increases accessibility to scientifically interesting areas and makes a safe landing. Vision-based navigation, ${ }^{1)}$ which estimates state such as position and velocity of a spacecraft by comparing maps with landmarks in images of a planetary surface, is one of the guidance, navigation, and

(c) 2020 The Japan Society for Aeronautical and Space Sciences

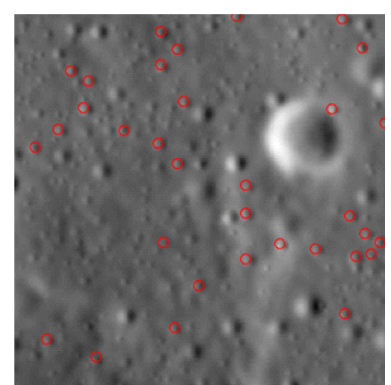

(a) Lunar surface image.

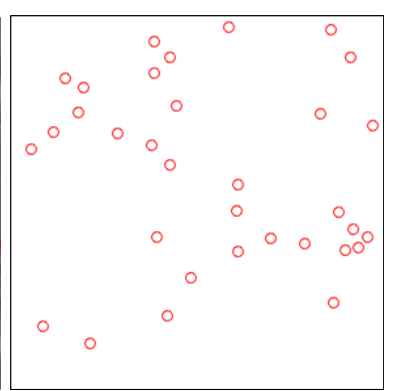

(b) Detected craters.
Fig. 1. Images of crater detection. The left shows lunar surface image taken by a spacecraft. Red circles show position of craters. The right shows detected position of craters.

control functions to achieve high precision landing. The typical vision-based navigation technologies involve image processing tasks such as crater detection ${ }^{2)}$ (see Fig. 1), matching, ${ }^{3)}$ feature tracking, etc. Realization of vision-based navigation on celestial bodies with gravity and long distance from the earth requires onboard and real-time image processing without communicating with ground stations because of high-speed descent and communication propagation delay.

However, despite the demand for fast image processing tasks, space-grade computers perform worse than commercial ones. The performance degradation is mainly due to the inability to clock up caused by thermal constraints, and the acquisition of radiation resistance. In the past decade, non-von Neumann type processor chips such as field-programmable gate arrays (FPGAs) are expected to improve the processing speed by parallel processing. The FPGAs are becoming mainstream of the image signal processors on spacecraft. However, at present, the spacegrade processors lag behind by 1-2 orders of magnitude in the 
performance compared to the commercial ones. ${ }^{4)}$ As future exploration missions make a more advanced landing to farther celestial bodies, power consumption requirements for processors become severer, and navigation algorithms become more complicated. Therefore, it is essential to bridge the gap between the complexity of algorithms and the performance of processors in terms of low power consumption.

Recently, several studies have addressed asynchronous computing systems mimicking neurobiological architecture like our brain called neuromorphic computing. In the brain, communication between the neurons is achieved by asynchronous transmitting trains of action potentials (spikes) to the subsequent neurons. Because these individual spikes are sparse in time, each spike has a high amount of information. Some research projects $^{5-8)}$ have developed the neuromorphic processor chips that compute with the low power consumption and high speed by imitating such dynamics of the spiking neurons. While conventional processors compute sequentially, the neuromorphic processors compute parallelly by asynchronous and sparse signals. The neuromorphic operation based on a spiking neural network (SNN) reduces calculation time and power consumption. The recent demonstration ${ }^{9)}$ of the neuromorphic processor shows that convolutional neural networks (CNNs) with a million spiking neurons on the chip can be operated with only a few tens of milliwatts of power consumption.

The success of research on such neuromorphic processors brings the possibility that advanced vision-based navigation algorithms such as deep learning can work with the spacecraft's low power computing environment. Although the effectiveness of neuromorphic computing for space systems is discussed, ${ }^{10}{ }^{1}$ no specific application exists. Therefore, this study proposes a method to convert artificial neural networks (ANNs) to spiking neural networks in order to operate neural network based visual navigation algorithms on the neuromorphic processor, and we also evaluate accuracy and power consumption using a crater classification task as a specific application for vision-based navigation.

The reminder of the paper is organized as follows: Components of an artificial neuron and a spiking neuron are outlined in section 2.1 and 2.2 and our method to convert ANN to SNN is presented in section 2.3. The results of simulating crater classification neural network converted to SNN are shown in section 3. The conclusion is given in section 4 .

\section{ANN-to-SNN Conversion Method}

Research on deep ANNs as a method of mimicking the computation of cranial nerves similar to SNNs has been remarkably successful in the field of computer vision. Although the ANNs are brain-inspired, there are some differences in their computation and learning rules compared to the SNNs. The SNNs operate asynchronously by the sparse spike current trains, so that the neuromorphic processors can be expected to have lower power consumption than conventional clock-based circuits. The dynamics of spiking neurons is time-dependent, while artificial neurons used for the ANNs only handle numerical values representing the firing rate of the neuron without the timing information. In order to operate ANN-based applications on the neuromorphic processors, it is necessary to bridge the gap be- tween the timing-based spiking neurons and the rate-based artificial neurons and to develop methods to make an SNN with equivalent functions as an ANN.

This section describes how to convert ANNs using a rectified linear unit (ReLU) activation function into SNNs. First, mechanisms of the artificial neuron and the spiking neuron are described. Our method to convert the ANNs into the SNNs using modified ReLU and transfer learning is following.

\subsection{Artificial neuron}

In fully connected deep networks widely used as deep ANNs, all neurons in the preceding layer are entirely connected to the subsequent layer without connecting inside the layer. The artificial neurons work by applying a linear summation of inputs and weights to nonlinearity. ReLU is a type of activation functions with nonlinearity and is used in many networks because of effectiveness to accuracy improvement. The computation of the fully connected networks using ReLU is described by

$$
\begin{aligned}
x_{i} & =\operatorname{ReLU}\left(0, \sum_{j} w_{i j} x_{j}+b_{i}\right) \\
& =\max \left(0, \sum_{j} w_{i j} x_{j}+b_{i}\right),
\end{aligned}
$$

where $x_{i}$ is the activation of neuron $i, w_{i j}$ is the weight connecting neuron $j$ in the preceding layer to neuron $i$ in the current layer, $x_{j}$ is the activation of neuron $j$ in the preceding layer, and $b_{i}$ is the bias term of neuron $i$. The output neurons of the network are activated by propagating successively updating all activations of the current layer to the subsequent layer.

$\mathrm{CNN}^{17)}$ is feed-forward network architecture in which feature detectors take convolution filters. This network model consists of alternating convolutional layers and pooling layers and finally activates the output neurons through fully connected layers. The convolutional layer generates feature maps by convolving the preceding layer with a kernel of arbitrary size. The resulting feature maps using ReLU as activate function are given by

$$
x_{i}^{k}=\max \left(W^{k} \otimes x^{l}+b^{k}\right),
$$

where $x_{i}^{k}$ is the resulting activation $i$ of the feature map of kernel $k, W^{k}$ is one of a set of convolutional kernels, the $\otimes$ denotes a two-dimensional convolution operator, $x^{l}$ is the patch $l$ of the preceding layer's activations, and $b^{k}$ is the bias term of kernel $k$.

The pooling layer following the convolutional layer has a function of subsampling the feature map input from the preceding layer. Typically, max-pooling is used to calculate this layer to reduce the computational complexity and to achieve good accuracy, as is described by

$$
x_{i}=\max \left(x^{l}\right)
$$

Although the accuracy is lower compared to the maxpooling, ${ }^{18)}$ some tasks often use average pooling given by

$$
x_{i}=\overline{x^{l}} .
$$


Input

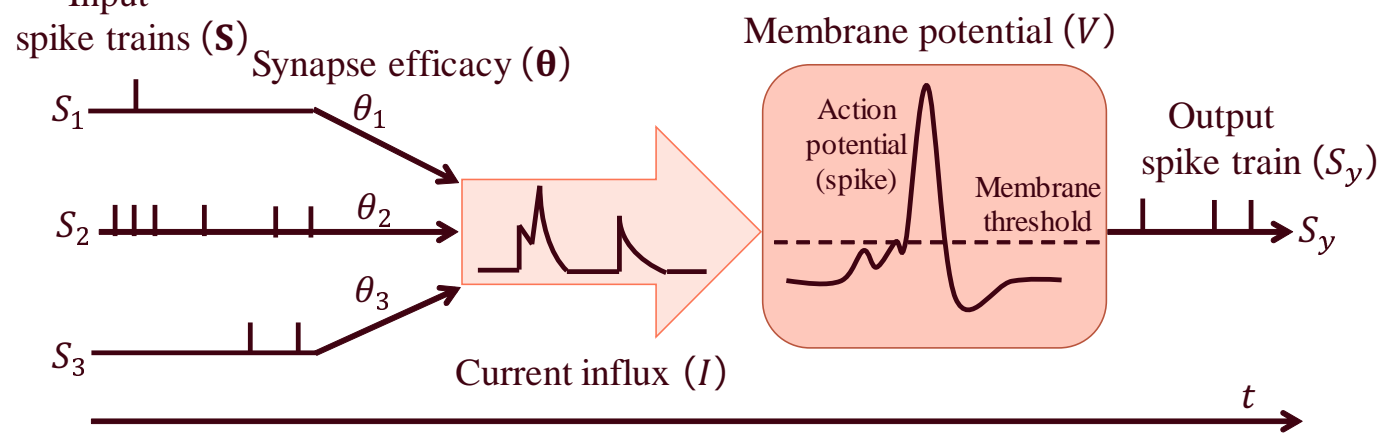

Fig. 2. A spiking neuron. Spike trains are input to the neuron as linear summed current with synaptic efficacy, then the neuron generates an output spike when the membrane potential reaches the threshold.

By alternately processing the convolutional layer and the pooling layer, CNNs reduce the dimensionality of the data, which is less likely to cause over-fitting problem because the amount of the weight parameters is smaller compared to the fully connected network. Besides, feature extraction by twodimensional convolution is suitable for image input. Therefore, many image recognition tasks use the $\mathrm{CNN}$ as feature extractor and detector.

\subsection{Spiking neuron}

The traditional ANNs communicate using continuous valued activations between the layers. In contrast, sparse sequences of events are input in SNNs. Spiking neurons integrate them to generate spikes for signaling in the preceding layers to drive spike firing of the final output neurons. Due to this temporally sparse spike events, the SNNs provide energy efficient operation. A schematic diagram of the spiking neuron used in the SNN is shown in Fig. 2.

The artificial neuron takes numerical values as input and works as a weighted linear summation followed by the activation function, as shown in Eq. (1). The inputs of the spiking neuron are sparse spike trains, where the spikes follow a Poisson distributed with a constant firing rate. When a spike reaches a post-synaptic neuron, the spike is input to the neuron as an electric current having an amplitude called synaptic efficacy and decaying exponentially. The current pulses consequently drive neuron's membrane potential to change over time and generate spikes when the membrane potential reaches the threshold. As above, the SNN operates asynchronously by sparse spike current, so that neuromorphic processors mimicking the SNN operation can be expected to have lower power consumption than the conventional clock-based circuits.

There are several models representing the neuronal dynamics, and a leaky integrate-and-fire (LIF) neuron is used in general neuromorphic processors. ${ }^{7,8)}$ The LIF neuron model dynamics is given by

$$
\tau_{m} \frac{d V(t)}{d t}=-V(t)+I(t)
$$

where $\tau_{m}$ is the membrane time constant, $V(t)$ is the membrane potential, and $I(t)$ is the input current. When the potential reaches a normalized threshold of the membrane potential $V_{t h}=1$, the neuron fires a spike, and the potential is held at zero for a refractory period $\tau_{r}$. In order to consider the spiking neurons as an activation function of the ANN, Eq. (5) needs to be deformed to represent a response function, i.e. firing rate $\lambda$.
Given a constant input current $I(t)=J$, Eq. (5) can be solved for the time, and the firing rate of the LIF neuron is given by

$$
\lambda(J)=\left\{\begin{array}{ll}
{\left[\tau_{r}-\tau_{m} \log \left(1-\frac{V_{t h}}{J}\right)\right]^{-1}} & \left(J>V_{t h}\right) \\
0 & \text { (otherwise) }
\end{array} .\right.
$$

If the input spike train has the constant current, it is possible to approximate the firing rate of the spiking neuron with sufficient accuracy by training ANN using Eq. (6) instead of an activation function. However, in practice, the input current is not constant because noise is generated due to the randomness of the spike trains. Also, since the derivative of Eq. (6) approaches infinity near the origin, it is difficult to use an ANN-specific learning rule such as backpropagation.

To address the issue, Refs. 14) and 15) perform that approximate the firing rate of the LIF neurons containing noise and learn a deep belief network by the Contrastive Divergence algorithm. In Ref. 16), CNNs are learned by back propagation by modifying the firing rate of the LIF neurons to a differentiable form. The modified LIF neuron is described by

$$
\lambda(J)=\left[\tau_{r}+\tau_{m} \log \left(1+\frac{V_{t h}}{\gamma \log \left(1+e^{\frac{J-V_{t h}}{\gamma}}\right)}\right)\right]^{-1}+\eta(J),
$$

where

$$
\eta(J)=\left\{\begin{array}{ll}
\operatorname{Gauss}(0, \sigma) & \left(J>V_{t h}\right) \\
0 & (\text { otherwise })
\end{array},\right.
$$

and $\gamma$ is an adjustable parameter to have control over the amount of smoothing. Smaller $\gamma$ makes Eq. (7) closer to the response function of LIF neuron. By learning the ANN using the response function of approximated LIF neuron instead of ReLU, it is possible to bring the weight parameters of the network closer to the synaptic efficacy of SNN.

Most CNNs reduce complexity by spatially downsampling feature maps using max-pooling. To achieve this in spiking neurons, computing the maximum firing rate of spike trains is necessary, but this computation for the asynchronous spikes is nontrivial. Suggestions for expressing max-pooling with spiking neurons using lateral inhibition ${ }^{11)}$ or time-to-first-spike encoding $^{12)}$ do not select the actual maximum firing rate, so they can not replace for max-pooling properly. Instead, average pooling can be easily converted to spike domain by changing the 
weights of the neuron. In this paper, average pooling is used to properly convert the representation of ANN to SNN.

\subsection{Conversion method from ANN to SNN}

The ability of neural networks to recognize visual patterns or to understand information is a result of several processing steps and learning mechanisms. In comparison to the artificial network, the concept of training methods of the spiking network is in its early phases. A straightforward approach to make applications work with SNNs is to convert pre-trained ANN parameters to the SNN with equivalent architecture. Such studies on so-called offline learning ${ }^{11,13)}$ achieved character classification tasks by SNNs with good accuracy by using weight normalization and a conversion scheme that utilizes a response function to be associated with activation of ReLU. Although their method achieves good performance with error rate in the ANN conversion, constraints are added in spiking neuron models, activation functions, pooling layers, and network architecture. The next approaches ${ }^{14-16)}$ show how to transfer weights directly to target SNN by training ANN whose activation functions are modified to a form suitable for spiking neurons. This method has generality in that input data, and weight parameters do not have to be post-processed. Although these approaches achieved good results on MNIST dataset, ${ }^{17)}$ actual image classification tasks are not discussed. Table 1 shows recent models for converting ANN to SNN. This table shows applicable network types, spiking neuron types and training methods used in the conversion models.

As above, it is necessary to convert the learning result of the ANN to the SNN to execute an application of the ANN in the SNN due to lack of effective learning rules. In order to evaluate the applicability of an image navigation task to the neuromorphic processors, our method replaces the CNN activation functions with modified LIF neurons and transfers the trained parameters directly to SNN for inference. The procedure of our conversion method is as follows:

1. Train an original feed-forward ANN.

2. Re-train the network with modified neurons with layer architecture equivalent to Step 1 using transfer learning.

3. Transfer the trained weight parameters in Step 2 directly to the SNN with layer architecture equivalent to Step 1.

In Step 1, the original ANN to be converted is trained as usual. In this study, the conversion is performed on a deep ANN of the four-layer architecture (784in-20conv-20pool-2fc) that performs a crater classification task ${ }^{19,20)}$ described later. In Step 2, re-training the network is carried out. It is with the activation function replaced by modified LIF response function given by Eq. (7) and the pooling layer modified from max-pooling to average pooling. In addition to the modification, transfer learning is performed using the weight parameters of Step 1 to improve the convergence and accuracy of re-training. Finally, in Step 3 , the inference is performed by directly transferring the weight parameters learned in Step 2 to the SNN with the same structure as Step 1.

\section{Simulation Results}

This section describes results of simulation experiments to verify the conversion method. First, detail of crater classifica-

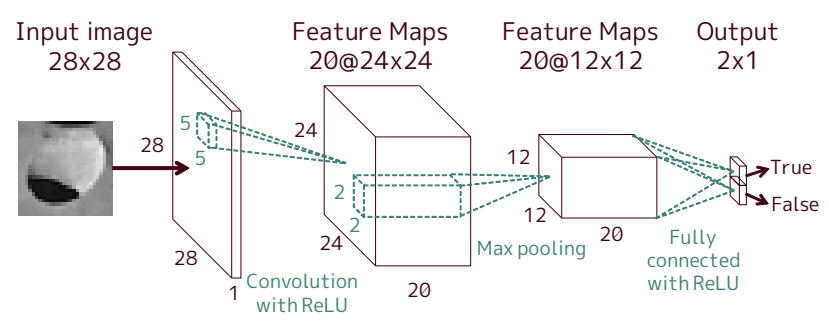

(a) Original deep ANN architecture trained in step 1 of the conversion method. It contains $28 \times 28$ input units, followed by convolution and maxpooling layers with $20 @ 5 \times 5$ convolutional kernel and $2 \times 2$ pooling window, and two output units fully connected to the preceding layer.

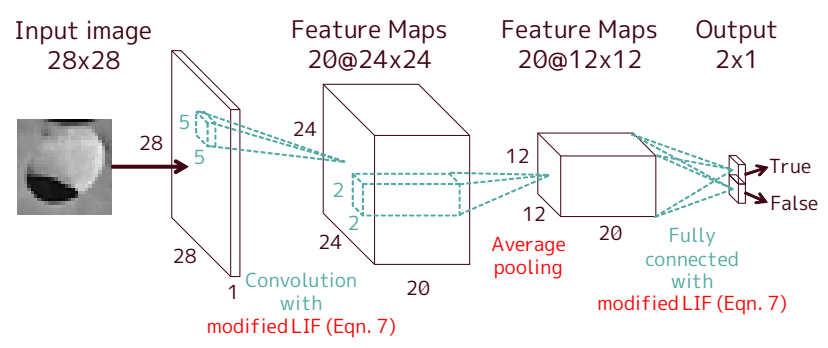

(b) Modified deep ANN architecture trained in step 2 of the conversion method. The network's neurons were changed from activation functions of all units to the response function of LIF neurons and the max-pooling to average pooling.

Fig. 3. Architecture of tested ANN.

tion ANN that is used in simulation and experimental environment is described. The results of simulation and energy analysis is following.

\subsection{Experimental description}

The crater classification network ${ }^{19,20)}$ was converted to a spiking network and trained 30,000 crater/non-crater images. The architecture of the network to train is shown in Fig. 3(a). This network takes a $28 \times 28$ one channel image as input and performs convolution using $205 \times 5$ kernels in the subsequent layer, and then subsampling is performed by max-pooling with two strides of $2 \times 2$ pooling window. Finally, two output units are fully connected to the preceding feature maps to represent the classified crater.

First, the original network was trained according to Step 1 of the conversion procedure described in Section 2.3. Next, Transfer learning of the modified network was performed to estimate the parameters of the spiking network. Figure 3(b) shows the modified network architecture used in Step 2. In this network, the ReLU function used as the activation function in the convolution layer and the fully connected layer of the original network is replaced with the response function of the modified LIF neuron shown by Eq. (7). Also, max-pooling was replaced with average pooling to decrease the complexity of the SNN. Both of these networks were trained using backpropagation and conjugate gradient method.

Parameters used in the modified LIF neuron $\tau_{r}, \tau_{m}, \gamma$ and $\sigma$ have a large influence on the success of the training. The key to setting the parameters is to reduce differences between the ReLU used in the ANN and the LIF used in the SNN. In particular, the derivatives of the nonlinearity for inputs need to be around one like ReLU, and the smoothing around the origin 
Table 1. Summary of recent SNN models.

\begin{tabular}{|c|c|c|c|}
\hline Model & Network type & Neuron type & Training method \\
\hline $\mathrm{Cao}^{11)}$ & Spiking CNN & Integrate-and-fire & Offline learning \\
\hline Diehl $^{13)}$ & Spiking CNN & Integrate-and-fire & Offline learning and weight normalization \\
\hline O'connor ${ }^{14)}$ & Spiking deep belief network & Leaky integrate-and-fire & Contrastive divergence \\
\hline Stromatias ${ }^{15)}$ & Spiking deep belief network & Leaky integrate-and-fire & Contrastive divergence, hardware \\
\hline Hunsberger ${ }^{16)}$ & Spiking CNN & Leaky integrate-and-fire & Direct conversion \\
\hline This paper & Spiking CNN & Leaky integrate-and-fire & Direct conversion with transfer learning \\
\hline
\end{tabular}

Table 2. Parameter setting for the modified LIF neurons using simulation.

\begin{tabular}{cc}
\hline Parameters & Values \\
\hline$\tau_{r}$ & $1 \mathrm{~ms}$ \\
$\tau_{m}$ & $20 \mathrm{~ms}$ \\
$\gamma$ & 0.02 \\
$\sigma$ & Eq. (9) \\
$\tau_{s}$ & $1 \mathrm{~ms}$ \\
\hline
\end{tabular}

needs to be close to the LIF curve. First, the membrane time constant $\tau_{m}$ was selected. $\tau_{m}=20 \mathrm{~ms}$ was selected because training in step 2 failed with a large value $(40 \mathrm{~ms})$ and a small value $(10 \mathrm{~ms})$. Due to the dense input data, $\tau_{r}=1 \mathrm{~ms}$ was selected to be small enough in a biologically plausible range to reduce the saturation effects on neuronal behavior. Smaller the smoothing parameter $\gamma$ makes the nonlinearity closer to the LIF curve. $\gamma=0.02$ was selected because training failed with a smaller value (0.01). Finally, the variance of the current of neuron $j$ is given ${ }^{21)}$ by

$$
\sigma_{i}^{2}=\frac{1}{2} \tau_{s} \sum_{j} w_{i j} \lambda_{j},
$$

where $\tau_{s}$ is the synaptic time constant. Smaller the parameter $\left(\tau_{s}=1 \mathrm{~ms}\right)$ was selected because the increased time taken for neurotransmitter release causes the mismatch between tha nonlinearity and LIF curve. Detailed sensitivity analysis may be possible if initial weights or combinations of these parameters are selected more judiciously. Table 2 shows the parameters for the modified LIF neuron using simulation (step 2).

The crater dataset for supervised learning was the same as that used in the original network. This dataset is a collection of lunar crater and non-crater terrain images with a size of $28 \times 28$ pix and contains about 30,000 training images which include scene under ideal and poor illumination conditions and craters having ideal and poor shape.

These images were cut out from images taken by the SELENE high definition camera system. ${ }^{22)}$

\subsection{Classification results}

The crater classification deep ANN was converted to the SNN using the conversion method described above and was run in simulation on a desktop computer with Intel i9-7980XE CPU and 64 GB RAM. MATLAB was used for the ANN training, and pyNN ${ }^{23)}$ and NEST simulator were used for the SNN simulation. The weight parameters were updated 128 images per batch and 20 epochs in conversion step 1 and 2, and the inference was performed at each conversion step for an input image of 8,000 cases.

Figure 4 provides examples of the input data used for the simulation. The top row shows crater/non-crater images used in training of the ANN, and the bottom row shows raster plots of Poisson spike trains as an input of the SNN generated by the top row images. The spike trains were propagated to subsequent layers by the dynamics of the spiking neuron described in section 2.2, then output at the last layer. The output firing rate and the output spike trains for which SNN was inferred by the input are shown in Fig. 5. The bottom raster plots show the output spike trains obtained by inferring the input images by the SNN, and the middle diagram shows the firing rate of the output spike trains. The vertical axis is the neuron index corresponding to the $2 \times 1$ output neuron in the network architecture shown in Fig. 3 , and the high firing rate expresses that the prediction probability is high. In the inference results of the input crater images (the 2nd, 4th, 5th, and 9th images from the left), the classification was correctly performed because their firing rates of the output neurons classified as the crater is high.

The classification accuracy in each step is shown in Table 3. The result shows that the proposed method can convert the original ANN to the SNN without significant loss of accuracy.

However, the classification accuracy of each step declined by $1.2 \%$ and $2.1 \%$. One cause of the accuracy loss is the mismatch due to replaces max-pooling with average pooling and differences between the modified activation function used in the modified network and the response function of the LIF neuron used in the spiking network. Incorrect classification cases were analyzed to find other causes.

Figure 6 shows examples of the incorrect cases for: (a) incorrect in the original network, (b) correct in the original network and incorrect in the modified network and (c) correct in the original network and the modified network and incorrect in the spiking network. And all incorrect cases in the upstream step were incorrect in the downstream step. The cases of (a) and (b) were composed of images with unclear crater rim and images with a little information due to shadows at almost the same ratio. Most cases of (c) were composed of images with high brightness and low contrast (92\% cases of (c)).

This shows that our modified network and spiking network failed to acquire feature representations of such inputs. Therefore, it is suggested that the accuracy may be improved by increasing the amount of training data or deepening the network in order to obtain more feature representations. However, the approachs need to be carefully determined because it makes training difficult and increasing the computational scale. In the spiking network, the result seems to indicate that dense and high-frequecy inputs across all neurons of the network cause the accuracy loss. Therefore, there is a possibility that accuracy is improved by inputting sparse data such as that acquired by a dynamic vision camera ${ }^{24)}$ suitable for the spike domain, instead of uniform and frequent spike input.

\subsection{Energy analysis}

One of the purposes of this research is to validate that the crater classification task in the SNN can be implemented on the 


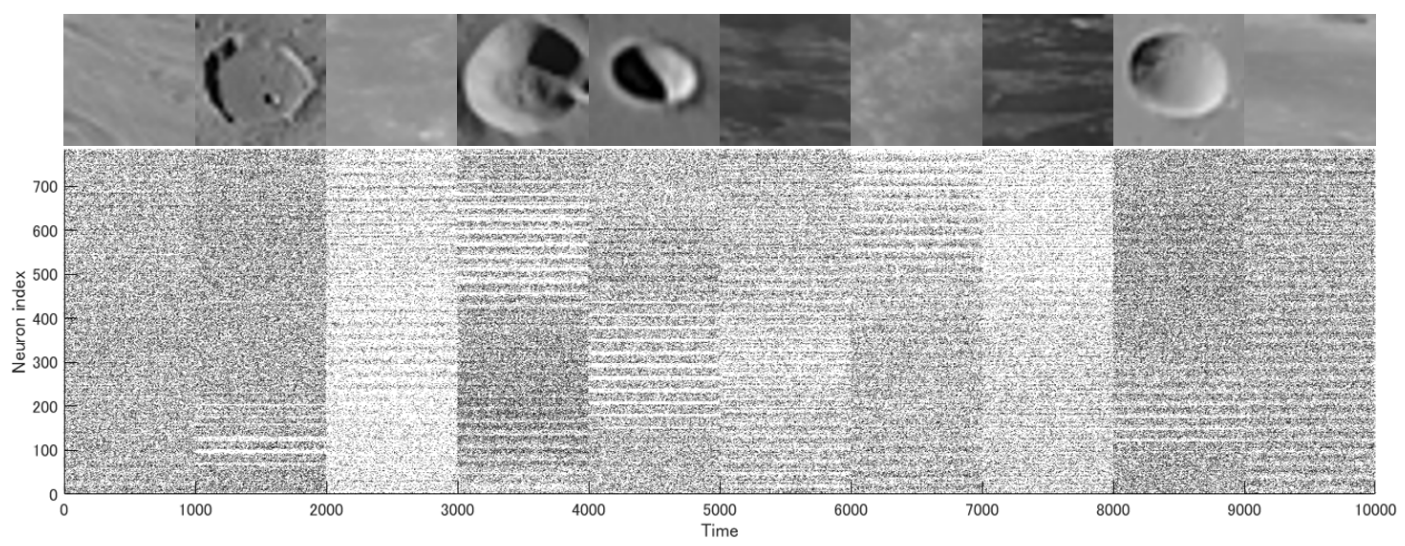

Fig. 4. Input data used for simulation experiments. The top images are crater/non-crater images input to ANNs (in Step 1, 2). The bottom diagram is raster plots of the $28 \times 28$ Poisson spike trains input to SNN generated from the top images.
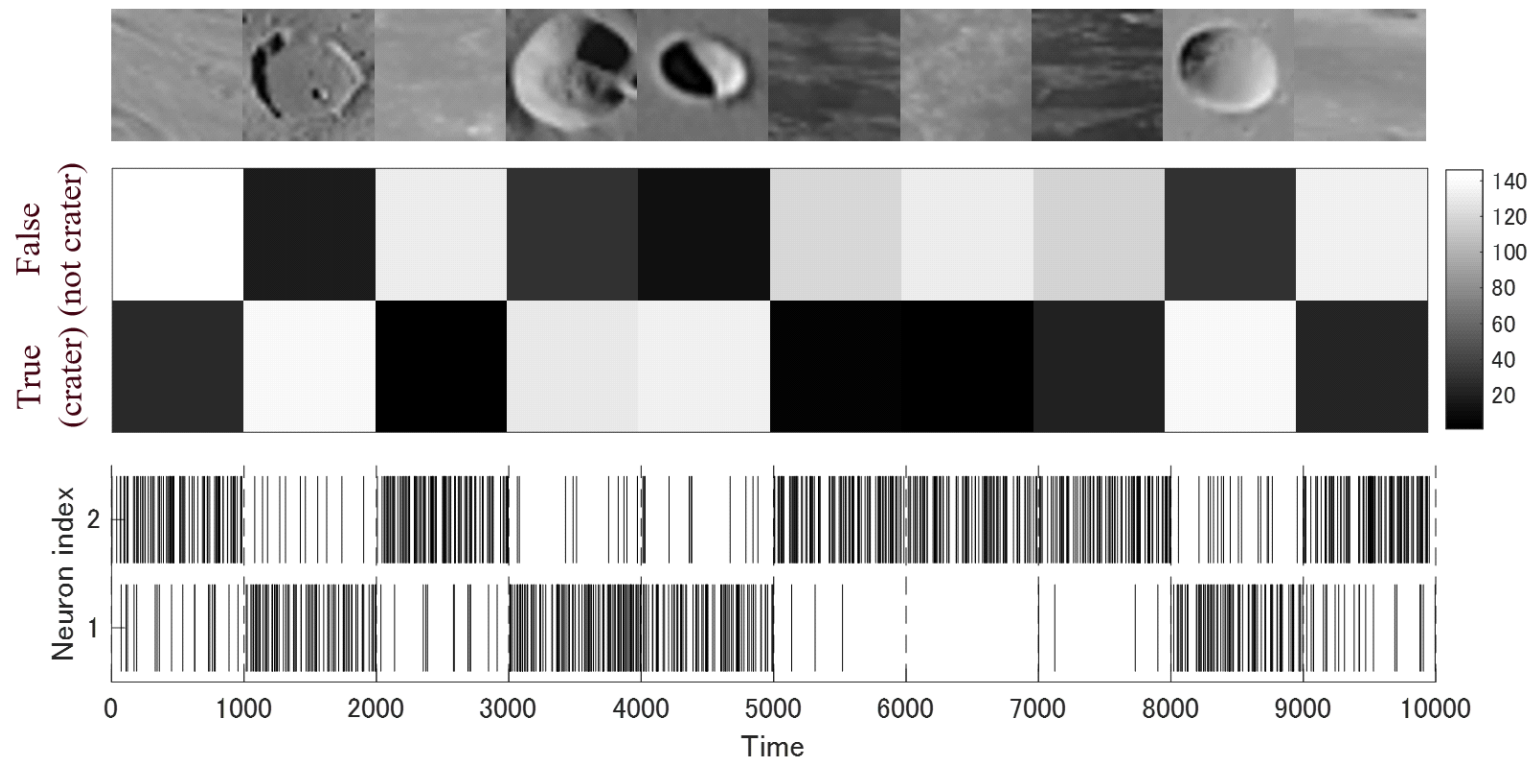

Fig. 5. Output results of the crater classification SNN converted by the method. The top row shows input images. The bottom diagram is raster plots of output spike trains for each input image, where the neuron index represents classification results. The middle figure shows firing rates of the output spike trains, and high firing rate represents that prediction probability is high.

Table 3. Classification accuracy in each step of the conversion method.

\begin{tabular}{cc}
\hline Step & Classification accuracy \\
\hline Original network & $99.4 \%$ \\
Modified network & $98.2 \%$ \\
Spiking network & $96.1 \%$ \\
\hline
\end{tabular}

neuromorphic processors with low power consumption. Energy consumption per spike is shown for some neuromorphic processors developed. ${ }^{6,25)}$ Based on the performance of such neuromorphic processors, we conduct an initial analysis of the power consumption of the crater classification task if the task is implemented in these processors. Reference 11) assumes that a spike consumes same energy, regardless of whether the spike is presynaptic or post-synaptic. We analyzed the power consumption of the neuromorphic processors based on that assumption.

For the analysis, the number of all spike activity in the neurons of the crater classification network was recorded at the simulation, and average 230 million spikes for processing an input image were measured during 1,000 milliseconds simulation time. Therefore, the energy consumed for processing each image by the neuromorphic processors is described by

$$
\begin{aligned}
E & =230 \times 10^{6} \text { spike/image } \times 1 \text { image } / \mathrm{s} \times \alpha \mathrm{pJ} / \text { spike } \\
& =230 \times 10^{6} \alpha \mathrm{pJ} / \mathrm{s} \\
& =230 \alpha \mu \mathrm{W},
\end{aligned}
$$

where $E$ is the total energy and $\alpha$ is the energy consumption of a neuromorphic processor. Estimates of power consumption of the crater classification SNN based on the performance of the typical neuromorphic processors using Eq. (10) is shown in Table 4.

Comparing power consumption is difficult, since it is highly dependent on implementation and network architecture. Nevertheless, it is worth to compare our results with previous reports, ${ }^{26,27)}$ which achieved power consumption of several to dozen watts with CNN implemented in FPGA having the performance equivalent to space-grade (Xilinx Virtex-7). Our model is similar to theirs in using $\mathrm{CNN}$ with convolutional layer and pooling layer as network architecture, but the computational scale and implementation method are notable different. The fact that our result is two orders of magnitude better in a rough comparison suggests that the SNN with the same func- 


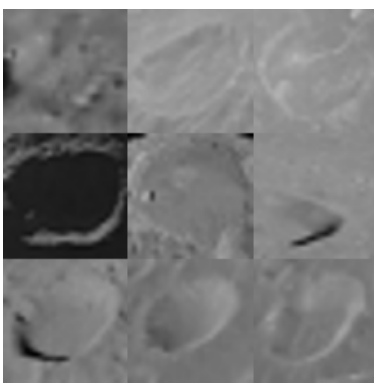

(a) Images incorrect in the origi- (b) Images correct in the original nal network.

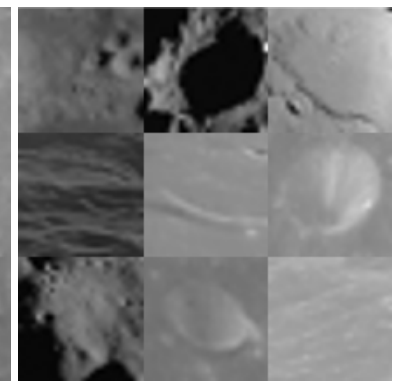
network and incorrect in the modified network.

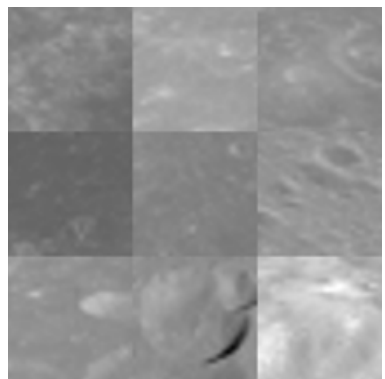

(c) Images correct in the original network and the modified network and incorrect in the spiking network.

Fig. 6. Examples of incorrect classification cases at each simulation step.

tionality as the ANN can be operated with ultra-low power consumption.

\section{Conclusion}

This study proposed a method to convert ANNs to SNNs in order to perform advanced vision-based navigation tasks with neuromorphic processors operating with low power consumption. In our approach, the original ANN is modified using an activation function appropriate to the spiking neuronal activity, and its architecture is re-trained using transfer learning. Then, SNN directly transferred the learned weight parameters can perform the same task as the original ANN. We applied the method to the crater classification ANN for verification of effectiveness. In the simulation experiment, the network consisting of four layers was trained by 30,000 crater/non-crater images and converted to the SNN then. The results show that our method can convert to the SNN without significant loss of the accuracy compared with the original ANN. In addition, power consumption for using neuromorphic processors to handle the crater classification task was analyzed briefly, and the result shows the network can be processed with a power consumption of only $0.085-10 \mathrm{~mW}$ per image using processors developed by Refs. 6) and 25).

The simulation results showed good performance, more detailed evaluations of power consumption, processing time, and mountability are necessary for performing on actual devices. Also, a method of generating sparse spike trains from input data to reduce spike activity is also important for power consumption and the processing time. When applying a neuromorphic application to an actual image navigation system, it is necessary to perform more complex tasks with SNN than the crater classification shown in this paper. Such tasks include crater detection,
Table 4. Estimates of the crater classification SNN power consumption mapping to neuromorphic processors.

\begin{tabular}{lll}
\hline Neuromorphic processors & $\alpha, \mathrm{pJ}$ & $E, \mu \mathrm{W}$ \\
\hline Merolla et al. (TrueNorth) $^{6)}$ & 45 & 10,350 \\
Cruz-Albrecht et al. $^{25)}$ & 0.37 & 85.1 \\
\hline
\end{tabular}

target tracking and optical flow estimation, and it is expected that efficient training and improved accuracy will be required for deeper and more complex network implementations. Further studies are needed in order to achieve these neuromorphic navigation systems.

\section{References}

1) Johnson, A. and Montgomery, J.: Overview of Terrain Relative Navigation Approaches for Precise Lunar Landing, 2008 IEEE Aerospace Conference, Montana, USA, pp. 1-10, 2008.

2) Okada, S., Nakahama, Y., Moribe, M., Kamata, H., Kariya, K., Takadama, K., et al.: Detection of the Position And the Size of Craters Using Principal Component Analysis and Its Evaluations, Aerosp. Tech. Jpn, 17 (2018), pp. 61-67 (in Japanese).

3) Kariya, K., Ishida, T., Sawai, S., Kinoshita, T., Kajihara, K., Iwasa, O., et al.: Position Estimation Using Crater-Based Linear Features for Pinpoint Lunar Landing, Aerosp. Tech. Jpn., 17 (2018), pp. $79-87$ (in Japanese).

4) Lentaris, G., Maragos, K., Stratakos, I., Papadopoulos, L., Papanikolaou, O., Soudris, D., et al.: High-Performance Embedded Computing in Space: Evaluation of Platforms for Vision-Based Navigation, J. Aerosp. Inform. Syst., 15 (2018), pp. 178-192.

5) Benjamin, B. V., Gao, P., McQuinn, E., Choudhary, S., Chandrasekaran, A. R., Bussat, J.-M., et al.: Neurogrid: A Mixed-AnalogDigital Multichip System for Large-Scale Neural Simulations, Proc. IEEE, 102 (2014), pp. 699-716.

6) Merolla, P. A., Arthur, J. V., Alvarez-Icaza, R., Cassidy, A. S. Sawada, J., Akopyan, F., et al.: A Million Spiking-Neuron Integrated Circuit with a Scalable Communication Network and Interface, Science, 345 (2014), pp. 668-673.

7) Friedmann, S., Schemmel, J., Grubl, A., Hartel, A., Hock, M., and Meier, K.: Demonstrating Hybrid Learning in a Flexible Neuromorphic Hardware System, IEEE Trans. Biomed. Circuits Syst., 11 (2017), pp. 128-142.

8) Furber, S. B., Galluppi, F., Temple, S., and Plana, L. A.: The SpiNNaker Project, Proc. IEEE, 102 (2014), pp. 652-665.

9) Esser, S. K., Merolla, P. A., Arthur, J. V., Cassidy, A. S., Appuswamy, R., Andreopoulos, A., et al.: Convolutional Networks for Fast, Energy-Efficient Neuromorphic Computing. Proc. Natl. Acad. Sci. U.S.A., 113 (2016), pp. 11441-11446.

10) Bersuker, G., Mason, M., and Jones, K. L.: Neuromorphic Computing: The Potential for High-Performance Processing in Space, The Aerospace Corporation's Center for Space Policy and Strategy, 2018, https://aerospace.org/paper/, (accessed June 27, 2019).

11) Cao, Y., Chen, Y., and Khosla, D.: Spiking Deep Convolutional Neural Networks for Energy-Efficient Object Recognition, Int. J. Comput. Vis., 113 (2015), pp. 54-66.

12) Orchard, G., Meyer, C., Etienne-Cummings, R., Posch, C., Thakor, N., and Benosman, R.: HFirst: A Temporal Approach to Object Recognition, IEEE Trans. Pattern Anal. Mach. Intell., 37 (2015), pp. 2028-2040.

13) Diehl, P. U., Neil, D., Binas, J., Cook, M., Liu, S.-C., and Pfeiffer, M.: Fast-Classifying, High-Accuracy Spiking Deep Networks through Weight and Threshold Balancing, 2015 International Joint Conference on Neural Networks, Killarney, Ireland, IJCNN-20157280696, 2015

14) O'Connor, P., Neil, D., Liu, S.-C., Delbruck, T., and Pfeiffer, M. Real-Time Classification and Sensor Fusion with a Spiking Deep Belief Networ Front. Neurosci., 7 (2013), 178.

15) Stromatias, E., Neil, D., Pfeiffer, M., Galluppi, F., Furber, S. B., and Liu, S.-C.: Robustness of Spiking Deep Belief Networks to Noise and 
Reduced Bit Precision of Neuro-Inspired Hardware Platforms, Front. Neurosci., 9 (2015), 114.

16) Hunsberger, E., and Eliasmith, C.: Spiking Deep Networks with LIF Neurons, arXiv preprint, arXiv:1510.08829, 2015 (accessed April 12, 2019).

17) LeCun, Y., Bottou, L., Bengio, Y., and Haffner, P.: Gradient-Based Learning Applied to Document Recognition, Proc. IEEE, 86 (1998), pp. 2278-2324.

18) Zhang, Y. and Wallace, B.: A Sensitivity Analysis of (and Practitioners' Guide to) Convolutional Neural Networks for Sentence Classification, 8th International Joint Conference on Natural Language Processing, Taipei, Taiwan, P2, 2017.

19) Ishida, T., Takahashi, M., and Fukuda, S.: Crater Detection Robust to Degradation and Illumination Changes Using Convolutional Neural Network, IEICE Technical Report, SANE2018-125, 2018 (in Japanese).

20) Ishida, T., Takahashi, M., and Fukuda, S.: Crater Detection Robust to Illumination and Shape Changes using Convolutional Neural Network, 32nd International Symposium on Space Technology and Science, Fukui, Japan, 2019-d-073, 2019.

21) La Camera, G., Giugliano, M., Senn, W., and Fusi, S.: The Response of Cortical Neurons to in Vivo-like Input Current: Theory And Experiment, Biol. Cybern., 99 (2008), pp. 279-301.
22) Yamazaki, J., Mitsuhashi, S., Yamauchi, M., Tachino, J., Honda, R., and Shirao, M.: High-Definition Television System onboard Lunar Explorer KAGUYA (SELENE) and Imaging of the Moon and the Earth, Space Science Reviews, 154 (2010), pp. 21-56.

23) Davison, A. P., Brüderle, D., Eppler, J., Kremkow, J., Muller, E. Pecevski, D., et al.: PyNN: A Common Interface for Neuronal Network Simulators, Front. Neuroinform. 2 (2009), 11.

24) Lichtsteiner, P., Posch, C., and Delbruck, T.: A 128x128 120dB 15 us Latency Asynchronous Temporal Contrast Vision Sensor, IEEE J. Solid State Circ., 43 (2008), pp. 566-576.

25) Cruz-Albrecht, J. M., Yung, M. W., and Srinivasa, N.: EnergyEfficient Neuron, Synapse and STDP Integrated Circuits, IEEE Trans. Biomed. Circuits Syst., 6 (2012), pp. 246-256.

26) Zhang, C., Li, P., Sun, G., Guan, Y., Xiao, B., and Cong, J.: Optimizing Fpga-based Accelerator Design for Deep Convolutional Neural Networks, 2015 ACM/SIGDA International Symposium on FieldProgrammable Gate Arrays, Monterey, USA, pp. 161-170, 2015.

27) Zong-ling, L., Lu-yuan, W., Ji-yang, Y., Bo-wen, C., and Liang, H.: The Design of Lightweight and Multi Parallel CNN Accelerator Based on FPGA, 2019 IEEE 8th Joint International Information Technology and Artificial Intelligence Conference, Chongqing, China, pp. $1521-1528,2019$ 